\title{
Enhancing HIV status disclosure and partners' testing through counselling in Tanzania
}

\author{
BONIPHACE IDINDILI', MAJIGE SELEMANI ${ }^{1}$, FAKIHI BAKAR ${ }^{1}$, SUMAIYYA G. THAWER ${ }^{2}$, ABDALLAH GUMI', \\ MWIFADHI MRISHO', AMOS M. KAHWA ${ }^{3}$ and JULIUS J. MASSAGA ${ }^{3}$ \\ 1lfakara Health Institute, Dar es Salaam, Tanzania \\ ${ }^{2}$ Imperial College, London, United Kingdom \\ ${ }^{3}$ National Institute for Medical Research, Dar es Salaam, Tanzania
}

\begin{abstract}
Background: In Tanzania HIV Testing and Counselling (HTC) is being implemented through voluntary counselling and testing (VCT), provider initiated counselling and testing (PITC) and work place counselling and testing (HTC). Within these programmes, HIV status disclosure is emphasized. However, among persons who test HIV positive, many do not disclose their status to their partners and social networks. However, data are lacking on the effectiveness of the different HTC strategies on HIV positive status disclosure.

Objective: To investigate which of the three HIV Testing and Counselling (HTC) strategies: Voluntary Counselling and Testing (VCT), Provider Initiated Counselling and Testing (PITC) and work place Counselling and testing is associated with improved HIV-positive status disclosure in Eastern Tanzania.

Methods: Structured interviews were conducted with 455 newly diagnosed HIV-positive clients at 6 HTC sites during enrolment and at three months follow-up to collect data on disclosure status.

Results: We found that PITC strategy attended a relatively higher proportion of clients $182 / 455(40.1 \%)$ as compared to VCT 169/455 (37.1\%) and work place HTC strategies 104/455(22.9\%) respectively. Among clients, about one third 130/455(28.6\%) were found to be HIV-positive. HIV status disclosure rates were variable and were in order of preference of disclosing to family members 86/130(66.2 \%), followed by relatives $74 / 130(56.9 \%)$ and sexual partners 71/130(54.6\%). A high proportion of participants $77 / 130(59.2 \%)$ experienced violence acts from sexual partners in form of stigma and discrimination, abuse, divorce and termination from employment. In the multivariate logistic regression, disclosure to sexual partners was associated with violence acts of about two times higher (Disclosure to Partners OR=1.89) when compared to the group that did not disclose to their partners.

Conclusion: PITC strategy was found to result into higher rates of HIV positive status disclosure when compared to VCT and work place HTC strategies. Stigma, discrimination and violence acts are still prevalent in Tanzania and discourages HIV positive status disclosure. Based on these findings, there is an urgent need of promoting public education on HIV transmission, prevention and treatment and enhancing strategies to reduce risky sexual behaviour and increase condom use.
\end{abstract}

Keywords: HIV, testing, counselling, disclosure, Tanzania

\section{Introduction}

Tanzania like other sub-Saharan African countries has the largest proportion of people infected with HIV/AIDS (MoHSW 2007; WHO 2006). The prevalence is estimated at $5.7 \%$ in the population segment aged 15-49 years old with women being highly infected than men (UNAIDS/WHO/UNICEF, 2013). The epidemic shows regional variations with urban residents having considerably higher infection levels at $10.9 \%$ when compared with peripheral residents 5.2\% (Somi et al. 2006; Mboera et al., 2015). HIV therefore poses as a major public health problem in Tanzania. In order to control and prevent HIV infections, successful strategies are required to prevent new infections and treat currently infected individuals (WHO, 2004). HIV testing and counselling (HTC) is a crucial way of promoting prevention, care and treatment. It serves as an important entity for achieving universal access to HIV services in many countries around the world (WHO, 2013). In Tanzania, HTC has been implemented mainly through Voluntary Counselling and Testing (VCT), Provider Initiated Testing and Counselling (PITC) and Work place HIV testing and Community-based HIV testing Campaigns (CBT). These strategies provide opportunities for HIV testing to individuals and serve as central points to HIV prevention. Within 
HTC programmes, emphasis is placed on the importance of HIV status disclosure among HIVinfected clients particularly to their sexual partners. It is well established that in order to achieve reduction in the incidence of HIV, disclosure of HIV status, which is an essential part of behaviour modification, is required (Norman et al., 2007). This is because knowing that a partner is HIVpositive is directly associated with condom use and inversely associated with multiple or casual sex partners (ljumba et al., 2004). In addition, HIV disclosure is central to HIV management especially for adhering to complex treatment regimens (Chesney et al., 1999; Norman et al., 2005). The benefits of HIV positive status disclosure extend further to a number of potential benefits for the individual including increased opportunities for social support, access to medical care and implementing HIV risk reduction with partners (Penelope, 2013).

Disclosure can be received by rejection and isolation resulting in an extremely stressful experience from partners, friends, family or the community (Paxton, 2002). Such experiences depend on individual's strategies to negotiate and counter the fear of disclosure (Norman, 2007). Individual's ability to make the choice of whether, how and to who to disclose is acquired during the process of HTC. Currently, the disclosure rate to sexual partners in Tanzania is still very low indicating the urgent need to "open up" the epidemic. A study by MacNeil et al. (1999) reported that after six months of diagnosis, the proportion of individuals who were most likely to share results with their spouses was only $24.7 \%$ and to sex partners was $18.8 \%$. Another study by Kilewo et al. (2001) reports lower disclosure rates to family members (5.6\%) as compared to sex partners (16.7\%) among women. Some other studies have attempted to measure disclosure at multiple assessment points. Antelman et al. (2001) found disclosure to sex partners increased from $22 \%$ within two months of diagnosis to $41 \%$ after about four years. MacNeil et al. (1999) reported that disclosure rates to sex partners increased from $5.8 \%$ to $18.8 \%$ after six months of diagnosis. In Tanzania, the rate of reported concealed HIV positive test results is higher (77.8\%) among urban HIV positive pregnant women than among their rural counterparts (18\%) (Mathews et al., 1999; Kilewo et al., 2001). Similar low disclosure rates have also been reported in other African countries including Burkina Faso, Rwanda and Kenya (WHO, 2011).

The public health importance of HIV positive status disclosure and sex partners HIV testing and counselling is three folds: (i) It provides opportunity for behaviour modification required to reduce the incidence of HIV; (ii) it promotes efforts in improving couple counselling and testing and linking them to ART services and other forms of support; and (iii) it improves access to medical care and increased adherence to medications. These public health goals can be met only through increased HTC for sex partners and disclosure of one's HIV positive status coupled with enhanced linkage of HIV positive persons to care and treatment services.

To-date, information on the impact of HTC strategy on HIV disclosure rates is limited. It is important that studies are required to describe the effects of HTC models on disclosure and sex partners testing and also assessing which model results in higher degree of disclosure, preserves the dignity and confidentiality of the affected person and increases awareness in the community about HIV/AIDS (UNAIDS/WHO, 2000). Such information is envisaged to help programmes on resource allocation, counselling of individuals who are not willing to disclose their HIV status and promoting sexual contact partner tracing. The objective of this study was to evaluate the effectiveness of VCT, PITC and Workplace HTC in HIV status disclosure in the subsequent three months after a HIV test.

\section{Materials and Methods}

\section{Study area}

The study was conducted at VCT centres, PITC health facilities and a Sugar cane farming company implementing workplace HTC that were situated five districts in the Eastern Zone of Tanzania. These were Bagamoyo (one urban and two rural facilities), Tanga (urban), Kilosa (rural) and 
Mvomero (in a rural located Mtibwa Sugar Estate). A convenient sampling approach has been used to select these study areas to ensure zonal coverage and cross-cultural representation. In addition, based on Tanzania Demographic and Health Survey of 2010, eastern zone of Tanzania had the highest number of people who know where to go for HIV testing compared to other zones in Tanzania.

\section{Study design}

This was a cross sectional study that utilized questionnaires for data collection at two time periods; at recruitment stage and at 3 months follow-up visits. Clients attending VCT, PITC and Workplace HTC programmes were recruited for the study. The inclusion criteria for participation required participants to be 13-49 years old and resident of the facility catchment area that provides HTC and voluntarily participation. Clients were consented to the study and underwent group and individual HTC and received disclosure counselling, partners testing and risk reduction information from counsellors. Individuals under 18 years old were consented under parental or guarding leadership.

\section{Data collection}

Data collection at first recruitment used a structured questionnaire to collect data on demographic characteristics, history of sexual behaviour, diagnosis of sexually transmitted infections and HIV status (intention to disclose) disclosure. Participants were asked to come back after 3 months and a brief structured questionnaire was administered to collect data on their disclosure experiences to their sex partners, close relatives, and social networks. Questionnaires were filled for each consenting patient and reviewed on a daily basis for validation of responses following interviews.

\section{Sampling and sample size}

The sample size required was calculated according to the following formula: $\mathrm{N}=\mathrm{t}^{2} \times \mathrm{p}(1-\mathrm{p}) / \mathrm{m}^{2}$ Where:

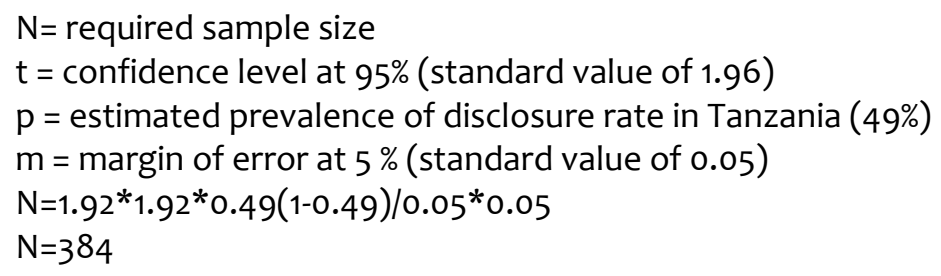

To correct for the difference in design, the sample size was multiplied by the design effect ( 1.5 based on WHO sample size calculation): $\mathrm{N}=384 * 1.5=576$. The sample was further increased by $5 \%$ to account for contingencies such as non-response or recording error: $(576 * 0.05)+576=605$ (including invited clients). The final sample size was therefore 605. There were 9 sites participating in this study and a minimum of 67 HIV positive clients were recruited from each HTC sites for this study. The sample size did not consider variations in the volume of HTC attendees.

\section{Data analysis}

All data was double checked for validation and cleaned and verified for any missing information according to protocol. A combination of variables were considered and analysed based on HTC strategy. Data were analysed using STATA software.

\section{Ethical considerations}

The study had scientific and ethical approval from the Ifakara Health Institute Review Board and the Medical Research Coordinating Committee of the National Institute for Medical Research. 
Permission was sought from Region, District and health facilities authorities. Written informed consent for participation was obtained from all study participants.

\section{Results}

\section{Baseline characteristics of participants}

A total of 600 subjects were enrolled into the study between March 2012 and August 2012. Out of these, 455 (75.8\%) participants provided complete baseline data for analysis and were therefore the first to be enrolled. A total of 145 (24.2\%) participants were registered after being invited for HTC but were excluded from the analysis because of insufficient information. Patients excluded did not differ from the remaining patients in terms of socio-demographic characteristics at the time of enrolment. The median age of participants was 30 years (range 13 to 70 years). Women were the majority of participants $(71.6 \% ; n=326 / 455)$. Slightly over half $(56.7 \% ; n=258 / 455)$ of the participants were married or cohabiting and a high proportion $311 / 455(80 \%)$ of the participants had primary school education (Table 1 ).

Table 1: Demographic characteristics of the study participants $(\mathrm{N}=455)$

\begin{tabular}{llll}
\hline Characteristics & & Number of respondents (\%) & 95\% CII \\
\hline Median age in years (range) & $30(13-70)$ & \\
Sex of respondent & Male & $129(28.4)$ & $18.1-41.6$ \\
& Female & $326(71.6)$ & $58.4-82.0$ \\
Marital status & Single & $146(32.1)$ & $23.9-41.6$ \\
& Married/Cohabiting & $258(56.7)$ & $44.5-68.1$ \\
\multirow{3}{*}{ Education level } & Divorced & $51(11.2)$ & $6.9-17.8$ \\
& No education & $53(11.6)$ & $5.8-22.0$ \\
& Primary & $311(68.4)$ & $56.7-78.1$ \\
Source of income & $\geq S e c o n d a r y$ & $91(20.0)$ & $12.9-29.7$ \\
& Not Employed & $261(42.6)$ & $19.3-69.8$ \\
\hline
\end{tabular}

Key: $\mathrm{Cl}=$ Confidence Interval

\section{HIV Testing and Counselling Strategy and HIV Test results}

Assessment of the HTC strategy used revealed that of the 455 participants, 169/455 (37.1\%), $182 / 455$ (40.1\%) and 104/455 (22.9\%) were HIV tested and counselled at VCT, PITC and Work place respectively (Table 2 ). Overall, 130/455(28.6\%) participants were HIV-tested positive and majority of them $94 / 182(52.7 \%)$ were attended at PITC sites. VCT participants had the lowest proportion of HIV-tested positive participants $18 / 169(10.7 \%)$. There is a strong association between type of HTC strategy and the HIV-positive results ( $p$-value $=0.001)$.

Table 2: Distribution of HIV test results by HTC Strategy $(\mathrm{N}=455)$

\begin{tabular}{llll}
\hline HIV Testing Strategy & No. of respondents (\%) & Positive (\%) & $\begin{array}{c}\text { HIV test status } \\
\text { Negative (\%) }\end{array}$ \\
\hline VCT & $169 / 455(37.1)$ & $18 / 169(10.7)$ & $151 / 169(89.4)$ \\
PITC & $182 / 455(40.0)$ & $94 / 182(52.7)$ & $88 / 182(48.4)$ \\
Workplace & $104 / 455(22.9)$ & $18 / 104(17.3)$ & $86 / 104(82.7)$ \\
Total & $455(100)$ & $130 / 455(28.6)$ & $325 / 455(71.4)$ \\
\hline
\end{tabular}

Between use strategy and HIV test results: Pearson chi2 $=80.552$, $p$-value $=0.001$

\section{Disclosure of HIV status}

Majority of participants $364 / 455(80 \%)$ expressed intention to disclose HIV test results to other people at recruitment while only $91 / 455(20 \%)$ of the participants were not willing to disclose their HIV status (Table 3 ). 
Table 3: Participants' intention to disclose HIV status by HTC strategy ( $\mathrm{N}=455)$

\begin{tabular}{llll}
\hline HIV Testing Strategy & Number of Respondents (\%) & \multicolumn{2}{c}{ Disclosure of HIV test result } \\
& & Yes (\%) & No (\%) \\
\hline VCT & $169 / 455(37.1)$ & $138 / 169(81.7)$ & $31 / 169(18.3)$ \\
PITC & $182 / 455(40.0)$ & $146 / 182(80.2)$ & $36 / 182(19.8)$ \\
Workplace & $104 / 455(22.9)$ & $80 / 104(76.9)$ & $24 / 104(23.1)$ \\
Total & $455(100)$ & $364 / 455(80)$ & $91 / 455(20)$ \\
\hline
\end{tabular}

Between use model and disclosure: Pearson chi2 $=0.911$, $p$-value $=0.795$

\section{Disclosure of HIV status and violence acts}

Analysis of the association of HIV-positive status disclosure to different social members and the occurrence of violence acts was conducted. The results showed that 86/130(66.2\%) HIV positive participants revealed their status to family members; $74 / 130(56.9 \%)$ revealed to relatives; $23 / 130(17.7 \%)$ revealed to friends; $27 / 130(20.7 \%)$ revealed to children and $71 / 130(54.6 \%)$ revealed to sexual partners. A total of $77 / 130(59.2 \%)$ HIV positive participants experienced one or more forms of violence acts after HIV-positive status disclosure. The violence acts reported included stigma and discrimination, fear, abuse, termination from employment and other actions.

The multivariate logistic regression revealed (Table 4) that disclosure to sexual partners was associated with violence actions of about two times higher (Disclosure to Partners OR=1.89) than the group that did not disclose to their partners. Disclosure to relatives was not likely to be received by violence acts $(\mathrm{OR}=0.16)$.

Table 4: Factors associated with violence acts when participants disclosed their HIV status ( $\mathrm{N}=130)$

\begin{tabular}{llll}
\hline HIV+ test Disclosure Status & $\begin{array}{l}\text { Multivariate analysis } \\
\text { Adjusted Odd ratio* }\end{array}$ & $\mathbf{9 5 \%} \mathbf{C l}$ & p-value \\
\hline Disclose to parents & 1.07 & $0.56-2.07$ & 0.816 \\
Disclose to children & 0.53 & $0.19-1.53$ & 0.212 \\
Disclose to relative & 0.16 & $0.07-0.37$ & 0.001 \\
Disclose to friends & 0.48 & $0.25-0.91$ & 0.028 \\
Disclose to partner & 1.89 & $0.69-5.16$ & 0.184 \\
Do not Disclose to any one & Reference* & & \\
\hline
\end{tabular}

* Reference being those clients who did not disclose to one of the factors

\section{Factors influencing HIV status disclosure}

Multivariate analysis was done to examine factors influencing HIV status disclosure. Strategies for HIV testing at VCT and workplace have significantly lower odds of HIV-positive participants disclosing their HIV status than PITC strategy (Table 5). When the age at first sex was less than 18 years, there was a significantly lower odds of HIV positive participants to disclose their HIV status (adjusted OR=0.27, 95\% Cl: 0.09-0.76) when compared to those who had first sex at age 18 to 24 years. This indicates that patients who became sexually active at less than 18 years were less likely to disclose their HIV status compared to patients who started sex at age 18 to 24 years. Patients who did not have STI were significantly less likely to disclose their HIV status compared to patients who were sick with STI. However, age of the respondent, educational level, sex, number of partners and HIV knowledge were not associated with HIV positive patients to disclose their HIV status.

In the univariate analysis, VCT, workplace, age at first sex less than 18 and patient not sick with STI had lower odds ratio of disclosing their HIV status (OR=0.16, $95 \% \mathrm{Cl}: 0.07-0.37$; OR=0.24, 
95\%Cl: 0.12-0.46; OR=0.35, 95\% Cl: $0.12-1.04$ and $\mathrm{OR}=0.63,95 \% \mathrm{Cl}: 0.42-0.96$ respectively). These variables were also statistically significant in multivariate model.

Table 5: Factors associated with HIV positive to disclose their HIV status $(\mathrm{N}=130)$

\begin{tabular}{|c|c|c|c|c|c|c|c|}
\hline \multirow[t]{2}{*}{ Factors } & & \multicolumn{3}{|c|}{ Univariate analysis } & \multicolumn{3}{|c|}{ Multivariate analysis } \\
\hline & & Odds ratio & $95 \% \mathrm{Cl}$ & P-value & $\begin{array}{l}\text { Adjusted } \\
\text { Odds ratio }\end{array}$ & $95 \% \mathrm{Cl}$ & p-value \\
\hline HIV Testing & PITC & Ref & & & & & \\
\hline \multirow[t]{2}{*}{ Strategy } & $\mathrm{VCT}$ & 0.16 & $0.07-0.37$ & 0.001 & 0.14 & $0.06-0.31$ & $<0.01$ \\
\hline & Workplace & 0.24 & $0.12-0.46$ & 0.001 & 0.15 & $0.07-0.30$ & $<0.01$ \\
\hline \multirow[t]{2}{*}{ Sex } & Male & Ref & & & & & \\
\hline & Female & 1.74 & $0.76-3.99$ & 0.164 & 1.49 & $0.63-3.51$ & 0.319 \\
\hline No. sexual & $2-10$ & Ref & & & & & \\
\hline \multirow[t]{2}{*}{ partners } & Less than 2 & 1.46 & $0.65-3.25$ & 0.314 & 1.66 & $0.75-3.66$ & 0.181 \\
\hline & Above 20 & 0.80 & $0.43-1.47$ & 0.425 & 1.02 & $0.30-3.41$ & 0.977 \\
\hline \multirow[t]{3}{*}{ Age at first sex } & $18-24$ & Ref & & & & & \\
\hline & Less than 18 & 0.35 & $0.12-1.04$ & 0.057 & 0.27 & $0.09-0.76$ & 0.019 \\
\hline & $25-30$ & 1.02 & $00.65-1.58$ & 0.939 & 1.30 & $0.87-1.94$ & 0.180 \\
\hline \multirow[t]{3}{*}{ Education level } & Primary & Ref & & & & & \\
\hline & $\geq$ Secondary & 0.80 & $0.32-2.03$ & 0.604 & 1.05 & $0.32-3.42$ & 0.933 \\
\hline & No education & 0.89 & $0.24-3.25$ & 0.842 & 1.94 & $0.56-6.71$ & 0.260 \\
\hline Age of the & $25-32$ & Ref & & & & & \\
\hline \multirow[t]{5}{*}{ respondents } & $18-24$ & 0.53 & $0.22-1.29$ & 0.140 & 0.47 & $0.18-1.23$ & 0.110 \\
\hline & Less than 18 & 0.81 & $0.35-1.89$ & 0.588 & 0.41 & $0.15-1.11$ & 0.073 \\
\hline & $33-40$ & 1.29 & $0.80-2.10$ & 0.257 & 1.24 & $0.77-2.00$ & 0.341 \\
\hline & $41-48$ & 0.59 & $0.30-1.19$ & 0.122 & 0.74 & $0.27-1.97$ & 0.498 \\
\hline & 49 and above & 0.55 & $0.10-3.06$ & 0.455 & 0.95 & $0.12-7.46$ & 0.960 \\
\hline \multirow[t]{3}{*}{ HIV knowledge } & Average & Ref & & & & & \\
\hline & Good & 1.04 & $0.51-2.13$ & 0.907 & 1.53 & $0.83-2.82$ & 0.149 \\
\hline & Poor & 0.59 & $0.20-1.73$ & 0.300 & 0.91 & $0.31-2.66$ & 0.842 \\
\hline \multirow[t]{2}{*}{ Sick of the STI } & Yes & Ref & & & & & \\
\hline & No & 0.63 & $0.42-0.96$ & 0.035 & 0.45 & $0.35-0.58$ & $<0.01$ \\
\hline
\end{tabular}

\section{Discussion}

This study found that PITC model attended a relatively high frequency of clients followed by VCT compared to the work place study model. This can be explained by the fact that majority of PITC clients are patients who have to take HIV test as part of their examination and supported by the expected assistance from family members and close relatives. These results provide evidence that PITC and VCT are important entry points to achieving a "universal access" to HIV services in Tanzania and thereby enhancing prevention, care and treatment of HIV (WHO, 2013).

Majority of participants in all three models expressed intention to disclose their HIV status after receiving test results. However, the disclosure rates were variable with preference of disclosing HIV status to family members, relatives followed by sexual partners. These disclosure rates are higher than the findings of a study by Kilewo et al. (2001), which reported lower disclosure rates to family members as compared to sex partners. Although disclosure rate was just above average (54.6\%) for sexual partners, results from FGD and in-depth interviews emphasize on the preference of HIV positive participants to disclose their status to family members and close relatives due to receiving of assistance in seeking medical care and financial support. Sexual partners responded negatively upon disclosure of HIV positive results by spouses. Violence acts were experienced by over half of the participants from sexual partners in the form of stigma and discrimination (community members), abuse, divorce and termination from employment. Some studies have shown that disclosure can be received by rejection and 
isolation resulting in an extremely stressful experience from partners, friends, family or the community (Paxton, 2002, Norman et al., 2005, 2007). Our results clearly support these findings by showing that HIV status disclosure is often met by violence acts by sexual partners and rejection by the community. This finding is important in considering the design of stigma and discrimination intervention strategies. Besides the benefits of assistance and support, disclosure was perceived as a medium to encourage family members and close relatives to accept HIV positive status, educate them on how to take care of themselves and help stop stigma and discrimination.

Our study did not find any motivation for individuals to take HTC. However in-depth interviews revealed that HTC during pregnancy (part of PITC) encourages uptake of HIV testing. Furthermore, we showed that education level and media did not contribute significantly in understanding HIV infection, prevention and care and promoting HIV public education. Rather much of the knowledge is acquired from health care workers during routine health talks. We also showed that condom use, which is a key method for HIV prevention, was only used by a small proportion of respondents. This poses a serious challenge to the HIV epidemic given that a large number of participants have multiple sexual partners, have high-risk sexual contact, report early sexual exposures and have history of STI in the past six months.

This study had some limitations. The study did not consider variability in the demographic profiles of categories HTC attendees, particularly the influence of employment for work place HTC uptake. In addition, inter-partner violence analysis was not stratified by gender. But also the fact that our sample size was calculated for descriptive study and not for hypothesis testing limited the generalization of the study findings. However the study managed to give a general view of the status of disclosure among communities of eastern Tanzania. We therefore encourage future studies to plan in advance for hypothesis testing with a priori sample size calculation for such a comparative study.

In conclusion, disclosure of HIV-positive status was received by violence and negative reactions by sex partners regardless of HTC strategy. These results call for an urgent need to increase public education on HIV transmission, prevention and care. This is crucial for eliminating stigma and discrimination to allow people living with HIV/AIDS to be accepted by their communities. All three HTC strategies are important entry points for HIV-infected people to receive care and treatment. The government should provide supportive systems to increase demand, access and utilization of these services.

\section{Acknowledgements}

We would like to acknowledge funding from the Global Fund Round 8 who funded this research project. We are grateful to all the study participants from all the participating sites of Makorora Health Centre, Chalinze health centre, Bagamoyo District Hospital, Ukuni VCT, Imani VCT, Mtibwa Hospital, Turiani Hospital and Kilosa District Hospital for their participation in this study. We further give thanks to all health workers of the participating sites who assisted in data collection and participants counselling and HIV testing.

\section{References}

Antelman, G., Smith Fawzi, M.C., Kaaya, S., Mbwambo, J., Msamanga, G.I., Hunter, D.J. \& Fawzi, W.W. (2001) Predictors of HIV-1 serostatus disclosure: a prospective study among HIVinfected pregnant women in Dar es Salaam, Tanzania. AIDS 15, 1865-1874.

Chesney, M.A. \& Ashley, W.S. (1999) Critical delays in HIV testing and care: the potential role of stigma. American Behavioral Scientist 427, 1162-1174. 
Ijumba, K., Gamieldien, R., Myer, L. \& Morroni, C. (2004) Sexual risk behaviours are influenced by knowing someone with HIV/AIDS. South African Medical Journal 94, 522-523.

Kilewo, C., Massawe, A., Lyamuya, E., Semali, I., Kalokola, F., Urassa, E., Giattas, M., Temu, F., Karlsson, K., Mhalu, F. \& Biberfeld, G. (2001) HIV Counselling and testing of pregnant women in sub-Saharan Africa: Experiences from a study on prevention of mother-to-child hiv-1 transmission in Dar es Salaam, Tanzania. Journal of Acquired Immune Deficiency Syndromes 28, 458-462.

MacNeil, J.M., Mberesero, F. \& Kilonzo, G. (1999) Is care and support associated with preventive behaviour among people with HIV? AIDS Care 11, 537-546.

Mathews, C., Kuhn, L., Frasman, D., Hussey, G. \& Dikwens, L. (1999) Disclosure of HIV Status and its consequences. South African Medical Journal 89, 1238.

Mboera, L.E.G., Ipuge, Y., Kumalija, J. Rubona, C.J., Perera, S., Masanja, H. \& Boerma, T. (2015) Mid-term review of national health plans: an example from the United Republic of Tanzania. Bulletin of the World Health Organization 93, 271-278.

Norman, A., Chopra, M. \& Kadiyala, S. (2005) HIV disclosure in South Africa: Enabling the Gateway to effective Response. International Food Policy Research Institute.

Norman, A., Chopra, M. \& Kadiyala, S. (2007) Factors related to HIV disclosure in 2 South African Communities. American Journal of Public Health 97, 1775-1781.

Paxton, S. (2002) The paradox of public HIV disclosure. AIDS Care 14, 559-567.

Penelope, T. (2013) Knowledge, attitudes and practices of HIV-positive patients regarding disclosure of HIV results at Betesda Clinic in Namibia. African Journal of Primary Health Care \& Family Medicine 5(1).

Somi, G.R., Matee, M.I.N, Swai, R.O., Lyamuya, E.F., Killewo, J., Kwesigabo, G., Tulli, T., Kabalimu, T.K., Ng'ang'a, L., Isingo, R. \& Ndayongeje, J. (2006) Estimating and projecting HIV prevalence and AIDS deaths in Tanzania using antenatal surveillance data. BMC Public Health 6: 120.

MoHSW (2007) National Guidelines for the Management of HIV and AIDS. National AIDS Control Programme, Ministry of Health and Social Welfare, $3^{\text {rd }}$ edition.

UNAIDS/WHO/UNICEF (2013) Epidemiology Fact Sheets on HIV and AIDS. UNAIDS/WHO/UNICEF.

WHO (2011) Women and Health: Today's Evidence Tomorrow's Agenda. World Health Organization. WHO (2006) WHO (2006) AIDS Epidemic Up-date. World Health Organization.

UNAIDS/WHO (2000) Opening up the HIV/AIDS epidemic. Geneva, Switzerland.

WHO (2004) Gender Dimensions of HIV status Disclosure to Sexual Partners. World Health Organization. 2004.

WHO (2013) HIV and Adolescents: Guidance for HIV testing and counselling and care for adolescents living with HIV. World Health Organization 2013. 